\title{
A New Memristor-Based 5D Chaotic System and Circuit Implementation
}

\author{
Rui Wang $\mathbb{D},,^{1,2}$ Mingjin Li $\mathbb{D},^{3}$ Zhaoling Gao $\mathbb{D}^{2},{ }^{2}$ and Hui Sun $\mathbb{D}^{2,4}$ \\ ${ }^{1}$ Tianjin Key Laboratory for Civil Aircraft Airworthiness and Maintenance, Civil Aviation University of China, Tianjin 300300, China \\ ${ }^{2}$ College of Information Engineering and Automation, Civil Aviation University of China, Tianjin 300300, China \\ ${ }^{3}$ Engineering Technology Department, Sichuan Airlines, Chengdu 610202, China \\ ${ }^{4}$ Department of Engineering Design and Mathematics, University of the West of England, Frenchay Campus, Coldharbour Lane, \\ Bristol, BS 161QY, UK \\ Correspondence should be addressed to Rui Wang; ruiwang@cauc.edu.cn and Hui Sun; h-sun@cauc.edu.cn
}

Received 30 June 2018; Revised 5 October 2018; Accepted 29 October 2018; Published 2 December 2018

Guest Editor: Viet-Thanh Pham

Copyright (c) 2018 Rui Wang et al. This is an open access article distributed under the Creative Commons Attribution License, which permits unrestricted use, distribution, and reproduction in any medium, provided the original work is properly cited.

\begin{abstract}
This paper proposes a new 5D chaotic system with the flux-controlled memristor. The dynamics analysis of the new system can also demonstrate the hyperchaotic characteristics. The design and analysis of adaptive synchronization for the new memristor-based chaotic system and its slave system are carried out. Furthermore, the modularized circuit designs method is used in the new chaotic system circuit implementation. The Multisim simulation and the physical experiments are conducted, compared, and matched with each other which can demonstrate the existence of the attractor for the new system.
\end{abstract}

\section{Introduction}

Memristors are the fourth kind of circuit elements except for resistors, capacitors, and conductors and are conceived by Chua in 1971 through the basic symmetric principle [1]. Furthermore, the corresponding theory was applied to memristive devices in 1976 [2]. It took a long time to develop the hardware memristor model. Until 2008 HP labs first realized the memristor of nanoscale in the form of crossbar array [3]. Since memristors have the potential applications in the wide range of fields, memristor study becomes hotter, and a huge amount of researchers have paid immense attention on memristor studies from industry and academics, respectively $[4,5]$. The typical examples include nonvolatile memories of nanoscale [6], memristor-based synapse in neuromorphic systems $[7,8]$, logic operations through material implication [9-12], and nonlinear dynamics in chaotic system [13-17].

With rapid development of memristor models, some studies combine the memristor and chaotic systems including dynamics analysis, image encryption applications and circuit implementations which have grown up quickly in recent years [18-22]. One of the typical early memristor-based chaotic systems was developed by Itoh and Chua in 2008 [19]. This paper developed some nonlinear oscillators by using memristors based on Chua’s oscillators. Petráš derived and investigated a fractional-order memristor-based Chua's circuit in [20]. Chua and Muthuswamy also discussed circuit topology and developed the simplest memristor-based circuits [21]. These papers demonstrate that the memristor oscillators own the special nonlinear dynamics due to memristors' extinguished characteristics. One of these significant characteristics is that the behaviors are dependent on initial states and circuit parameters. Li et al. proposed a scroll chaotic system circuit implementation by using HP memristor [22]. $\mathrm{Ma}$ et al. developed a four-wing hyperchaotic system by using a memristor adding over a three-dimensional chaotic system [16]. Dimitrios et al. found a new 4-D memristive chaotic system and investigated the behavior with hidden attractors of the system through numerical simulations [23]. Wang et al. proposed a flux-controlled memristor model and established a 4-D chaotic system with this model. The numerical analysis and circuit implementation simulation verification were conducted [24]. Mou et al. discussed the characteristics of dynamical behaviors of a fractional-order 
4D hyperchaotic memristive system and circuit simulation verification [25]. Other memristor-based hyperchaotic systems were also investigated such as numerical analysis about a four-dimensional hyperchaotic system with memristor and conducted circuit simulation verifications [26-29]. There are also some other chaotic systems developed by memristive models and its applications [30-32].

The above memristor-based chaotic system literatures focus on four or lower-dimensional chaotic systems and investigate the detailed numerical analysis and the corresponding numerical and circuit implementation simulation verifications. However, the higher-dimensional (5$\mathrm{D}$ or above) memristor-based hyperchaotic systems and the corresponding physical hardware experiments are not found. Therefore, the paper analyzes a new memristorbased hyperchaotic system and develops a circuit physical implementation method by using the modularized design method. This method is used to design the circuit without dimensions for chaotic circuit designs and is easy to be implemented in the circuit by using less circuit parts [33-38].

The novelty of this paper is to develop a new memristorbased 5D hyperchaotic system, design and analyze the adaptive synchronization of this new system, implement the physical experiment circuit hardware, and verify the existence of system attractors. The improved modularized design method is used to implement the circuit of the system to verify the existence of attractors.

The rest of the paper is organized as follows. Section 2 analyzes the fundamental characteristics of the new memristor-based chaotic system. Section 3 investigates the adaptive synchronization of the new memristor-based hyperchaotic system. Section 4 discusses the circuit implementation of the new system and verifies the existence of attractors. Conclusions are presented in Section 5.

\section{Analysis of a New Memristor-Based Hyperchaotic System}

In this section, numerical analyses are conducted for a fluxcontrolled memristor-based new 5D hyperchaotic system derived from Wang's 4D hyperchaotic system.

As illustrated in [16], memristor model is based on the fundamental characteristics of a flux-controlled memristor described below.

$$
i=W(\varphi) v
$$

where $i$ and $v$ are the current and the voltage of the device terminal, respectively. $W(\varphi)$ is the incremental memductance defined as

$$
W(\varphi)=\frac{d q(\varphi)}{d \varphi}
$$

This demonstrates that the characteristics of a memristor are a nonlinear function reflecting the relationship among the charge and flux across and through the device.
Furthermore, this paper consistently uses the smooth cubic monotone-increasing continuous nonlinearity described as follows [20-22, 33].

$$
d q(\varphi)=m \varphi+n \varphi^{3}
$$

where $m, n>0$.

Then the memductance is shown below

$$
W(\varphi)=\frac{d q(\varphi)}{d \varphi}=m+3 n \varphi^{2}
$$

This paper develops a 5D memristor-based chaotic system which is derived from four-wing autonomous chaotic dynamics systems reported by Wang et al. [34, 37]

$$
\begin{aligned}
& \dot{x}=a(y-x)+4 y z \\
& \dot{y}=-x+16 y-x z+w \\
& \dot{z}=-b z+x y \\
& \dot{w}=-10 y+0.15 x z \\
& \dot{u}=-x
\end{aligned}
$$

This system has four state variables $x, y, z$, and $w$, and $a, b \in$ $\mathbb{R}^{+}$.

Substitute (4) into (5), a 5D memristor-based system is obtained.

$$
\begin{aligned}
& \dot{x}=a(y-x)+4 y z-k x W(u) \\
& \dot{y}=-x+16 y-x z+w \\
& \dot{z}=-b z+x y-x u-y w \\
& \dot{w}=-10 y+0.15 x z-g z u \\
& \dot{u}=-x
\end{aligned}
$$

where

$$
W(u)=m+3 n u^{2}
$$

and $k, m, n, g$ are positive parameters.

2.1. Equilibria and Stability. The equilibrium points of System (6) can be calculated by solving the equations as shown below

$$
\begin{aligned}
a(y-x)+4 y z-k x W(u) & =0 \\
-x+16 y-x z+w & =0 \\
-b z+x y-x u-y w & =0 \\
-10 y+0.15 x z-g z u & =0 \\
-x & =0
\end{aligned}
$$

where $m=0.1, n=0.01$, and $g=0.3$.

System (6) has only one real equilibrium point with $(0,0$, $0,0,0)$ and has typical characteristics with a line equilibrium in $(0,0,0,0, \delta)$, given $\delta$ a real constant. 
First, analyze the zero equilibrium point $(0,0,0,0,0)$. The Jacobian matrix of System (6) on the zero equilibrium point is

$$
J_{0}=\left(\begin{array}{ccccc}
-a-0.1 k & a & 0 & 0 & 0 \\
-1 & 16 & 0 & 1 & 0 \\
0 & 0 & b & 0 & 0 \\
0 & -10 & 0 & 0 & 0 \\
-1 & 0 & 0 & 0 & 0
\end{array}\right)_{(0,0,0,0,0)}
$$

and the corresponding polynomial is

$$
f(\lambda)=\lambda(\lambda-b) f_{1}(\lambda)
$$

and

$$
\begin{aligned}
f_{1}(\lambda)= & \lambda^{3}+(a-16+0.1 k) \lambda^{2}+(10-15 a-1.6 k) \lambda \\
& +10 a+k
\end{aligned}
$$

It is obvious that 0 and $-b$ are eigenvalues of System(6) for the $(0,0,0,0,0)$ equilibrium point. According to RouthHurwitz condition, if and only if $a-16+0.1 k>0,10-$ $15 a-1.6 k>0,(10 a+k)>0$, and $(a-16+0.1 k)(10-$ $15 a-1.6 k)-(10 a+k)>0$ coexist, $f_{1}(\lambda)$ has the negative real number. However, the above four inequalities are not able to be realized simultaneously. Then not all real parts of the eigenvalues are negative. Therefore, it is not a stable equilibrium point.

Second, analyze the eigenvalues of Jacobian matrix of System (6) on the line equilibrium in $(0,0,0,0, \delta)$.

$$
=\left(\begin{array}{ccccc}
-a-k\left(0.1+0.03 \delta^{2}\right) & a & 0 & 0 & 0 \\
-1 & 16 & 0 & 1 & 0 \\
-\delta & 0 & -b & 0 & 0 \\
0 & -10 & -0.3 \delta & 0 & 0 \\
-1 & 0 & 0 & 0 & 0
\end{array}\right)
$$

Typically, when $a=14$ and $b=78, J_{1}{ }^{*}$ is calculated as shown below

$$
=\left(\begin{array}{ccccc}
-14-k\left(0.1+0.03 \delta^{2}\right) & 14 & 0 & 0 & 0 \\
-1 & 16 & 0 & 1 & 0 \\
-\delta & 0 & -78 & 0 & 0 \\
0 & -10 & -0.3 \delta & 0 & 0 \\
-1 & 0 & 0 & 0 & 0
\end{array}\right)
$$

Two of the five eigenvalues of $J_{1}{ }^{*}$ are complex conjugates; therefore, it is difficult to determine the stability of the line equilibria.
2.2. Symmetry. System (6) is symmetric with respect to $z$ axis since it is invariant when applying the coordinate transformations.

$$
(x, y, z, w, u) \longleftrightarrow(-x,-y, z,-w,-u)
$$

2.3. Dissipativity. Furthermore, dissipative characteristics analysis of System (6) is shown below. The system divergence is given by

$$
\begin{aligned}
\nabla V & =\frac{\partial \dot{x}}{\partial x}+\frac{\partial \dot{y}}{\partial y}+\frac{\partial \dot{z}}{\partial z}+\frac{\partial \dot{w}}{\partial w}+\frac{\partial \dot{u}}{\partial u} \\
& =-a-k W(u)+16-b \\
& =-k\left(m+3 n u^{2}\right)+16-a-b
\end{aligned}
$$

when $k>0, m>0, n>0,16-a-b<0,-k\left(m+3 n u^{2}\right)+16-$ $a-b<0$, System (6) is dissipative. The paper selects $m=0.1$, $n=0.01$ for equation (7), and $k=0.02$ for System (6).

2.4. Lyapunov Spectrum and Bifurcation Diagram. Fix parameters $a=14, b=78$ and vary the parameter $k$, and the graphs about the Lyapunov exponents versus $k$, bifurcation diagram, and phase portraits are shown in Figure 1.

Figure 1 shows that the Lyapunov exponents vary with the parameter $k$ changes. In the five Lyapunov exponents, three of them are obviously negative when $k \in[0,5.9]$. The top two lines in Figure 1(b) demonstrate that these two kinds of Lyapunov exponents are bigger than zero, and systems are hyperchaotic systems when $k$ lies in this range. In this paper, $k$ will be selected in this range.

When $a=14, b=78, k=0.02, m=0.1, n=0.01$, and $g=0.3$, the Lyapunov exponents are calculated as $L_{1}=1.0241$, $L_{2}=0.0137, L_{3}=-0.1735, L_{4}=-2.3787$, and $L_{5}=-70.3244$. This system is a hyperchaotic system with two positive Lyapunov exponents. Therefore, the Kaplan-Yorke dimension of System (6) can be found below

$$
D_{K Y}=4+\frac{L_{1}+L_{2}+L_{3}+L_{4}}{\left|L_{5}\right|}=3.978466
$$

\section{Adaptive Synchronization of a New Memristor-Based Hyperchaotic System}

In this section, the adaptive controller was designed for the new memristor-based hyperchaotic system which was derived by Lyapunov stability theory inspired by [39].

First, consider the master System (6) with $a=14, b=78$, $k=0.02, m=0.1, n=0.01$, and $g=0.3$ shown as below

$$
\begin{aligned}
& \dot{x}=14(y-x)+4 y z-k x\left(0.1+0.01 u^{2}\right) \\
& \dot{y}=-x+16 y-x z+w \\
& \dot{z}=-78 z+x y-x u-y w \\
& \dot{w}=-10 y+0.15 x z-0.3 z u \\
& \dot{u}=-x
\end{aligned}
$$




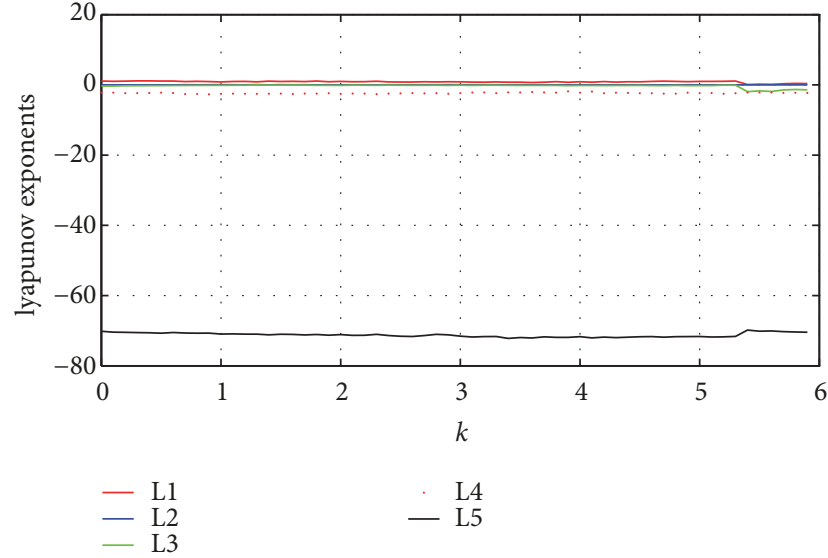

(a)

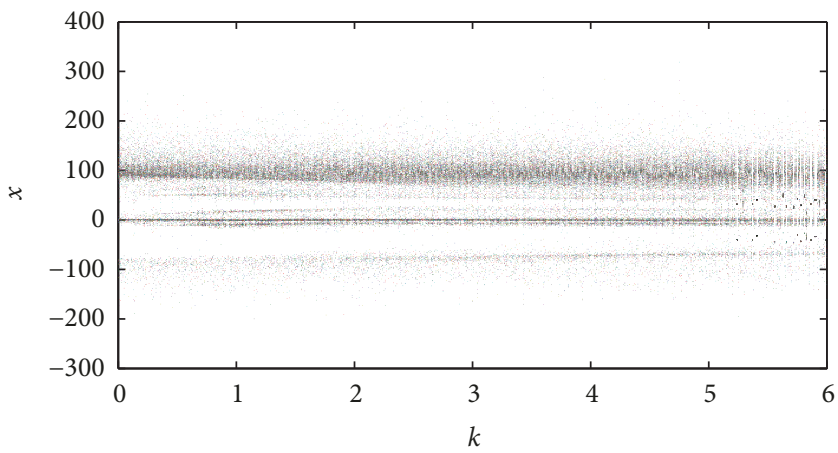

(c)

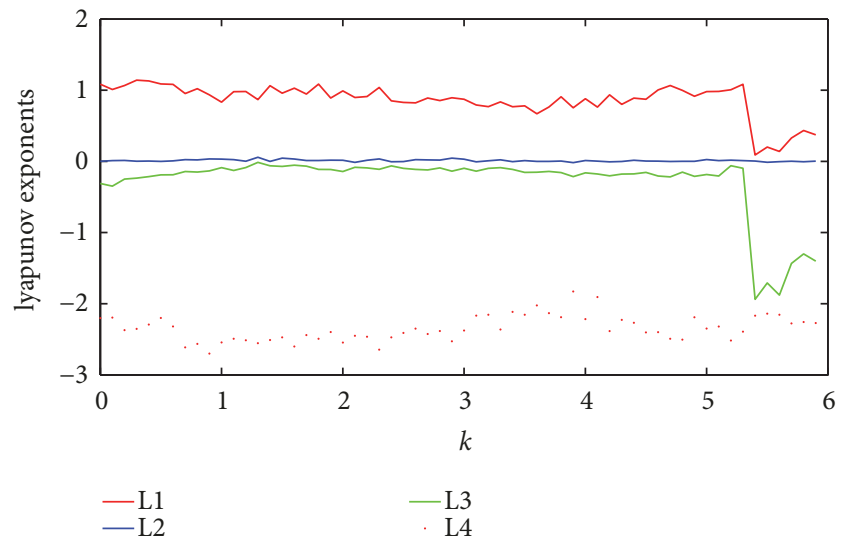

(b)

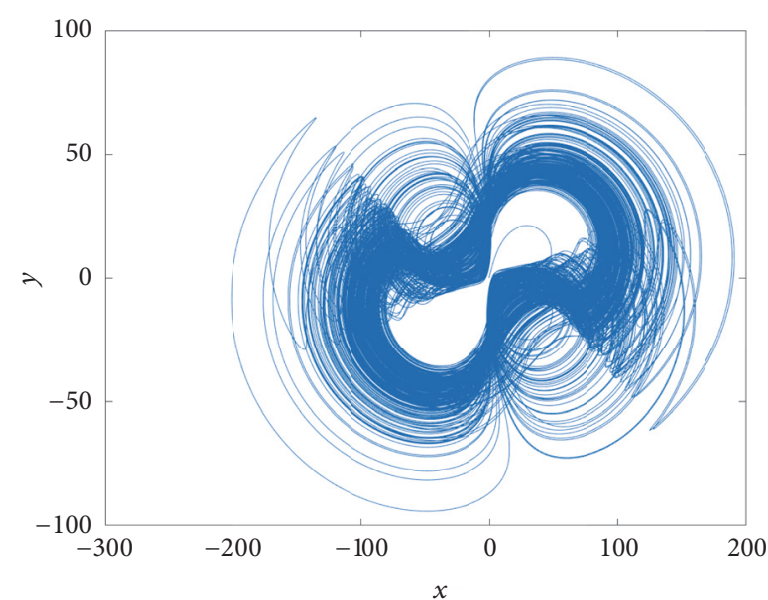

(d)

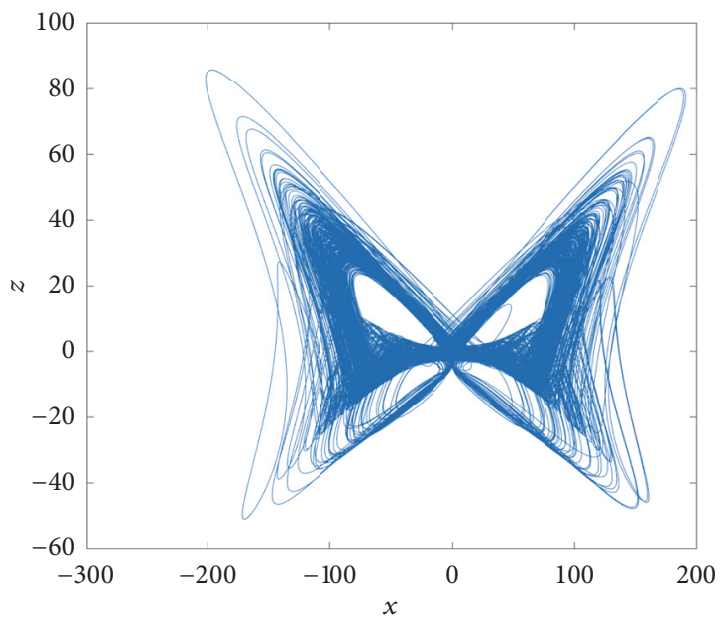

(e)

FIGURE 1: Lyapunov exponents versus the parameter $k$ : (a) the full five exponents, (b) Lyapunov exponents $L_{1}-L_{4}$ of System (6), (c) the bifurcation diagram of $x$ vs. $k$ for System (6) when $m=0.1, n=0.01$, and $g=0.3$. (d)-(e) The phase portraits $x-y$ and $x-z$ for System (6) when $k=0.02, m=0.1, n=0.01$, and $g=0.3$. 


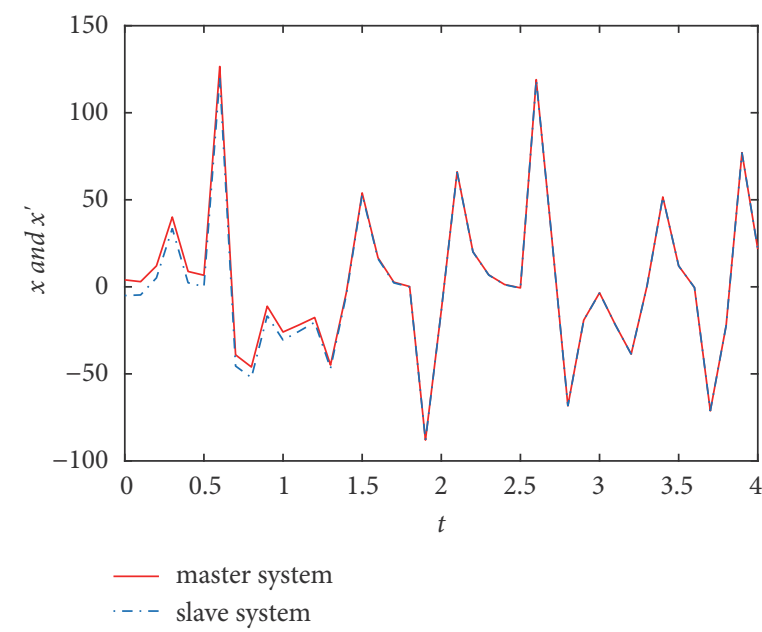

(a) Synchronization of states $x$ and $x^{\prime}$ for Systems (21) and (22)

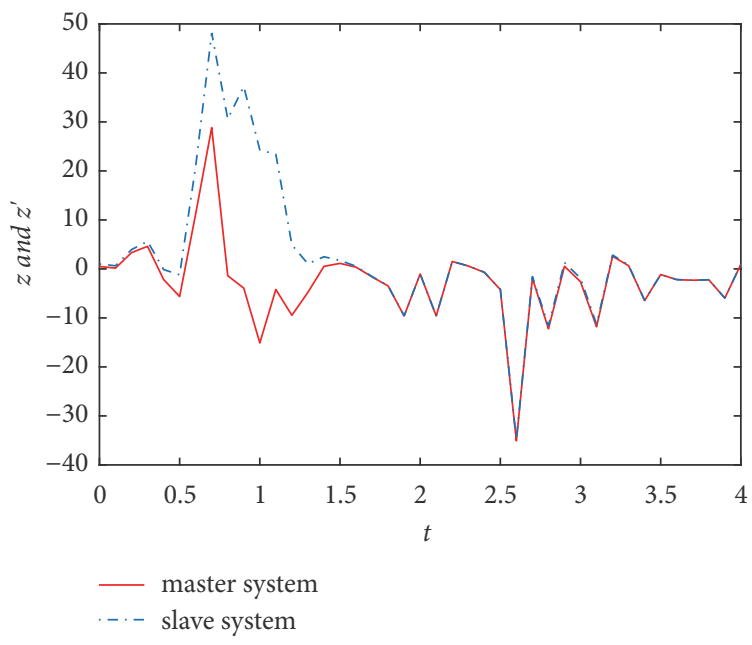

(c) Synchronization of states $z$ and $z^{\prime}$ for Systems (21) and (22)

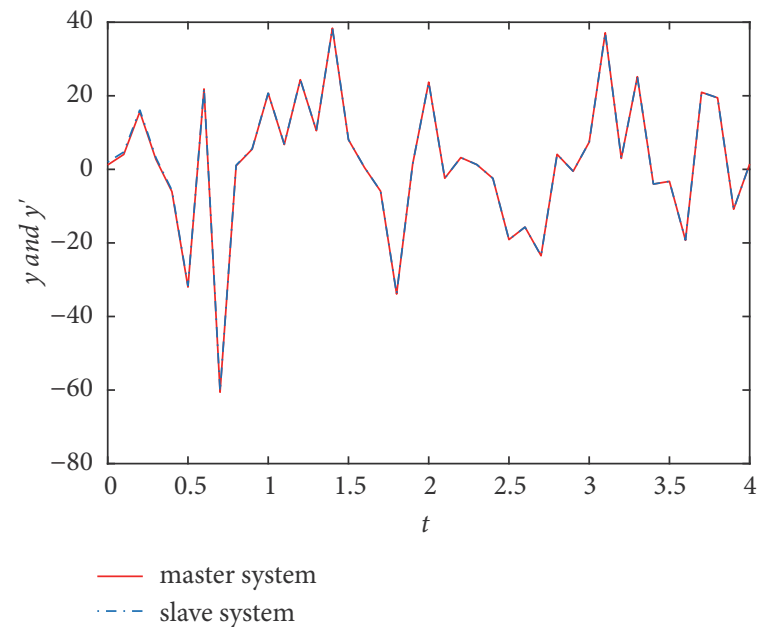

(b) Synchronization of states $y$ and $y^{\prime}$ for Systems (21) and (22)

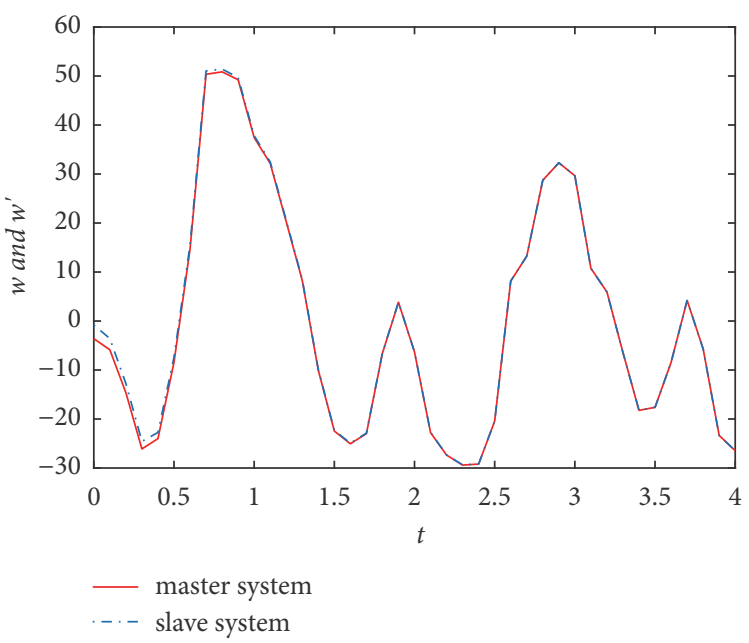

(d) Synchronization of states $w$ and $w^{\prime}$ for Systems (21) and (22)

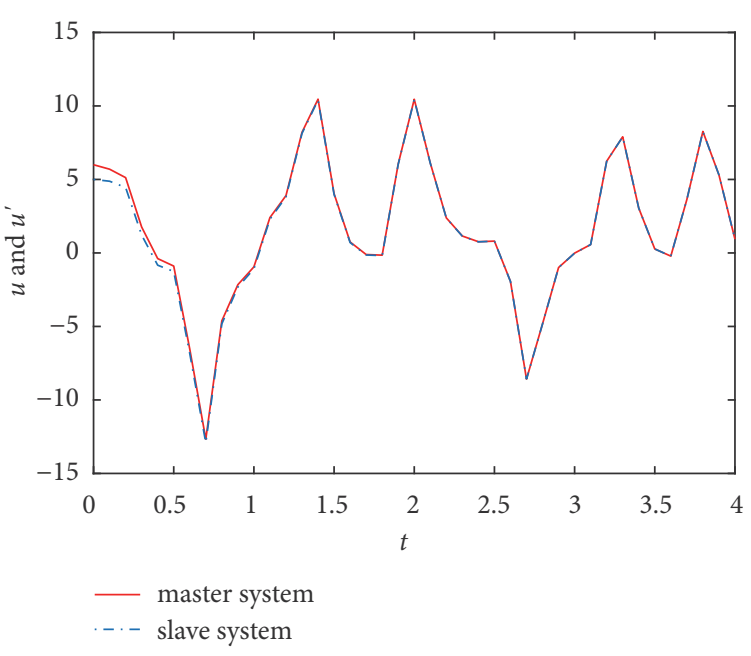

(e) Synchronization of states $u$ and $u^{\prime}$ for Systems (21) and (22)

FIGURE 2: Synchronization of states for Systems (21) and (22). 


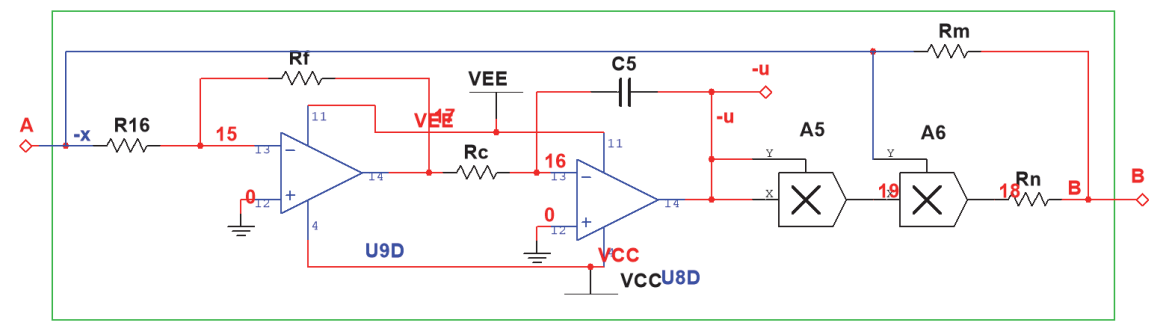

FIgURE 3: $W(u)$ function-memristor channel.

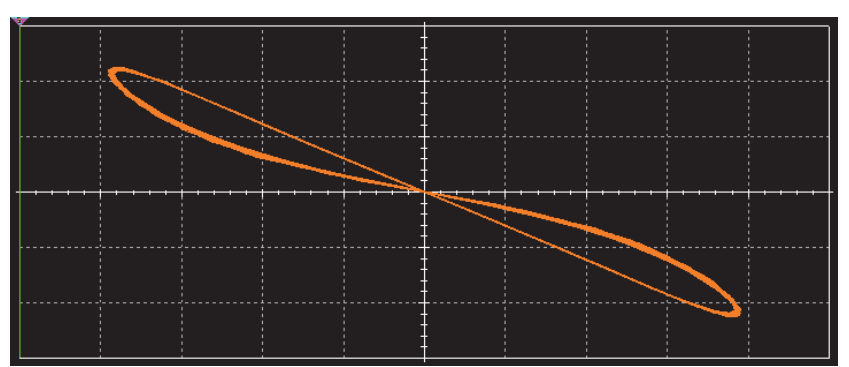

Figure 4: The relationship between $x$ and $W(x)$ for the circuit as shown in Figure 3.

Second, consider the slave system shown as below

$$
\begin{aligned}
& \dot{x}^{\prime}=14\left(y^{\prime}-x^{\prime}\right)+4 y^{\prime} z^{\prime}-k x^{\prime}\left(0.1+0.01 u^{\prime 2}\right)+u_{1} \\
& \dot{y}^{\prime}=-x^{\prime}+16 y^{\prime}-x^{\prime} z^{\prime}+w^{\prime}+u_{2} \\
& \dot{z}^{\prime}=-78 z^{\prime}+x^{\prime} y^{\prime}-x^{\prime} u-y^{\prime} w^{\prime}+u_{3} \\
& \dot{w}^{\prime}=-10 y^{\prime}+0.15 x^{\prime} z^{\prime}-0.3 z^{\prime} u^{\prime}+u_{4} \\
& \dot{u}^{\prime}=-x^{\prime}+u_{5}
\end{aligned}
$$

where $u_{i}(i=1 \cdots 5)$ are adaptive controller, and $x^{\prime}, y^{\prime}, z^{\prime}$, $w^{\prime}, u^{\prime}$ are the new state variables.

Therefore, the synchronization errors among the Systems (17) and (18) are defined as follows.

$$
\begin{aligned}
& e_{1}=x^{\prime}-x \\
& e_{2}=y^{\prime}-y \\
& e_{3}=z^{\prime}-z \\
& e_{4}=w^{\prime}-w \\
& e_{5}=u^{\prime}-u
\end{aligned}
$$

Then the dynamics of the error can be calculated by substituting the master and slave systems as shown below

$$
\begin{aligned}
\dot{e}_{1}= & a\left(e_{2}-e_{1}\right)-0.1 k e_{1}+4\left(y^{\prime} z^{\prime}-y z\right) \\
& -0.03 k\left(x^{\prime} u^{\prime 2}-x u^{2}\right)+u_{1} \\
\dot{e}_{2}= & -e_{1}+16 e_{2}+e_{4}-x^{\prime} z^{\prime}+x z+u_{2} \\
\dot{e}_{3}= & -b e_{3}+x^{\prime} y^{\prime}-x^{\prime} u^{\prime}-y^{\prime} w^{\prime}-x y+x u+y w+u_{3} \\
\dot{e}_{4}= & -10 e_{2}+0.15 x^{\prime} z^{\prime}-0.15 x z-0.3 z^{\prime} u^{\prime}+0.3 z u \\
& +u_{4} \\
\dot{e}_{5}= & -e_{1}+u_{5}
\end{aligned}
$$

where the adaptive controller $u_{i}$ are defined by

$$
\begin{aligned}
u_{1}= & -\widehat{a}(t)\left(e_{2}-e_{1}\right)+0.1 k e_{1}-4\left(y^{\prime} z^{\prime}-y z\right) \\
& +0.03 k\left(x^{\prime} u^{\prime 2}-x u^{2}\right)-k_{1} e_{1} \\
u_{2}= & e_{1}-16 e_{2}-e_{4}+x^{\prime} z^{\prime}-x z-k_{2} e_{2} \\
u_{3}= & \widehat{b}(t) e_{3}-x^{\prime} y^{\prime}+x^{\prime} u^{\prime}+y^{\prime} w^{\prime}+x y-x u-y w \\
& -k_{3} e_{3} \\
u_{4}= & 10 e_{2}-0.15 x^{\prime} z^{\prime}+0.15 x z+0.3 z^{\prime} u^{\prime}-0.3 z u \\
& -k_{4} e_{4} \\
u_{5}= & e_{1}-k_{5} e_{5}
\end{aligned}
$$

where $k_{i}(i=1, \ldots, 5)$ are the positive gains and $\widehat{a}(t), \widehat{b}(t)$ are the estimations of the corresponding parameters $a$ and $b$, respectively. into

Correspondingly, the dynamics of the error is changed

$$
\begin{aligned}
& \dot{e}_{1}=(a-\widehat{a}(t))\left(e_{2}-e_{1}\right)-k_{1} e_{1} \\
& \dot{e}_{2}=-k_{2} e_{2} \\
& \dot{e}_{3}=(b-\widehat{b}(t)) e_{3}-k_{3} e_{3} \\
& \dot{e}_{4}=-k_{4} e_{4} \\
& \dot{e}_{5}=-k_{5} e_{5}
\end{aligned}
$$




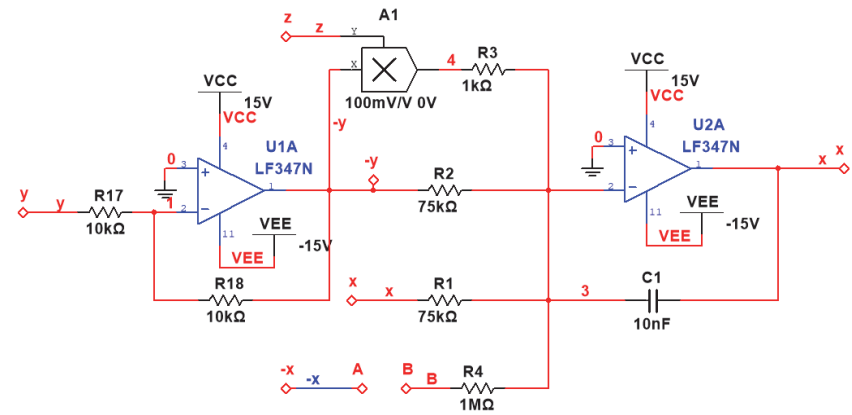

(a) $x$ channel

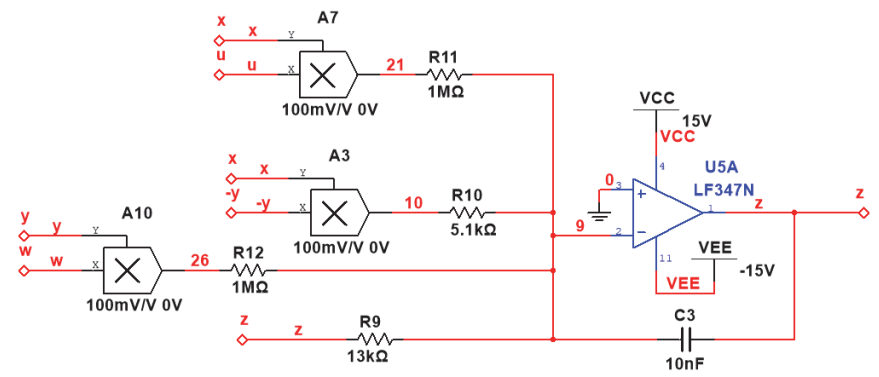

(c) $z$ channel

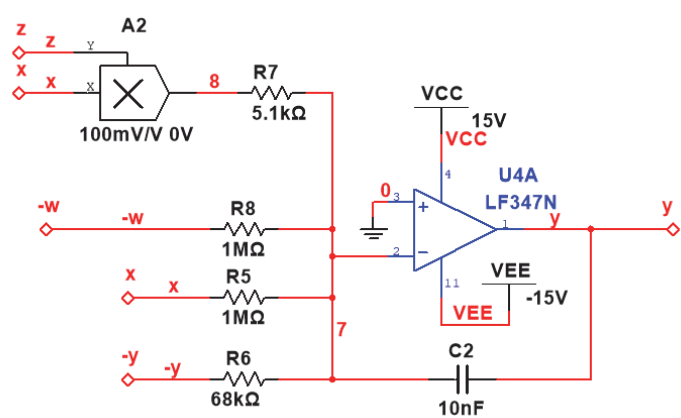

(b) $y$ channel

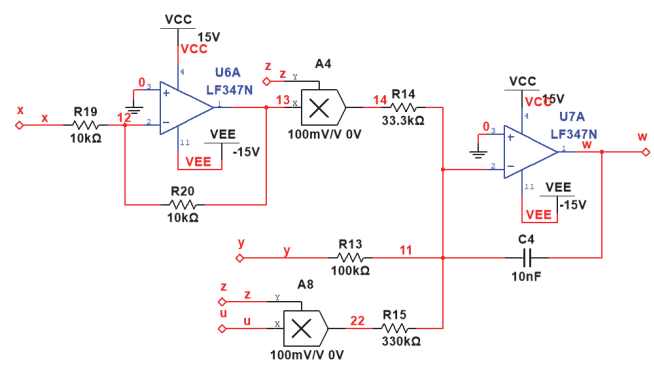

(d) $w$ channel

FIGURE 5: Modularized circuit channels for circuit implementation of the new memristor-based 5D hyperchaotic system.

Define the parameters for estimating errors as

$$
\begin{aligned}
& e_{a}(t)=a-\widehat{a}(t) \\
& e_{b}(t)=b-\hat{b}(t)
\end{aligned}
$$

Substitute (23) into the dynamics (22), the simplified error dynamics is described as

$$
\begin{aligned}
& \dot{e}_{1}=e_{a}(t)\left(e_{2}-e_{1}\right)-k_{1} e_{1} \\
& \dot{e}_{2}=-k_{2} e_{2} \\
& \dot{e}_{3}=-e_{b}(t) e_{3}-k_{3} e_{3} \\
& \dot{e}_{4}=-k_{4} e_{4} \\
& \dot{e}_{5}=-k_{5} e_{5}
\end{aligned}
$$

Correspondingly,

$$
\begin{aligned}
& \dot{e}_{a}(t)=-\widehat{a}(t) \\
& \dot{e}_{b}(t)=-\widehat{b}(t)
\end{aligned}
$$

Depending on the master and slave systems definitions and error dynamics transformations mentioned above, consider a Lyapunov function defined by

$$
V=\frac{1}{2}\left(e_{1}^{2}+e_{2}^{2}+e_{3}^{2}+e_{4}^{2}+e_{5}^{2}+e_{a}^{2}+e_{b}^{2}\right)
$$

Differentiate $V$ along the trajectories for equations (24)-(25), $\dot{V}$ can be obtained as

$$
\begin{aligned}
\dot{V}= & e_{1} \dot{e}_{1}+e_{2} \dot{e}_{2}+e_{3} \dot{e}_{3}+e_{4} \dot{e}_{4}+e_{5} \dot{e}_{5}+e_{a} \dot{e}_{a}+e_{b} \dot{e}_{b} \\
= & -k_{1} e_{1}^{2}-k_{2} e_{2}^{2}-k_{3} e_{3}^{2}-k_{4} e_{4}^{2}-k_{5} e_{5}^{2} \\
& +e_{a}\left[-e_{1}^{2}-e_{1} e_{2}-\dot{\hat{a}}(t)\right]-e_{b}(t)\left[e_{3}^{2}+\dot{\vec{b}}(t)\right]
\end{aligned}
$$

Then, the parameter update law is

$$
\begin{aligned}
& \dot{\vec{a}}(t)=-e_{1}^{2}-e_{1} e_{2} \\
& \dot{\hat{b}}(t)=-e_{3}^{2}
\end{aligned}
$$

Theorem 1. The master and slave Systems (17)-(18) for a new memristor-based hyperchaotic system along with unknown parameters and positive gains $k_{i}(i=1 \cdots 5)$ synchronize exponentially by using the adaptive controller (21) and the parameter update law (28).

Proof. Substitute the parameter update law (28) into the adaptive controller (21), then

$$
\begin{aligned}
\dot{V} & =-k_{1} e_{1}^{2}-k_{2} e_{2}^{2}-k_{3} e_{3}^{2}-k_{4} e_{4}^{2}-k_{5} e_{5}^{2} \leq-k^{\prime}\|e\|^{2} \\
& \leq 0
\end{aligned}
$$

where $k^{\prime}=\min \left\{k_{1}, k_{2}, k_{3}, k_{4}, k_{5} \mid k_{i} \in \mathbb{R}^{+}, i=1 \cdots 5\right\}$.

It is obvious that $e(t) \longrightarrow 0$ exponentially as time goes to infinite for all initial conditions of $e(0)$. According to Lyapunov stability theory, two systems are synchronized. 


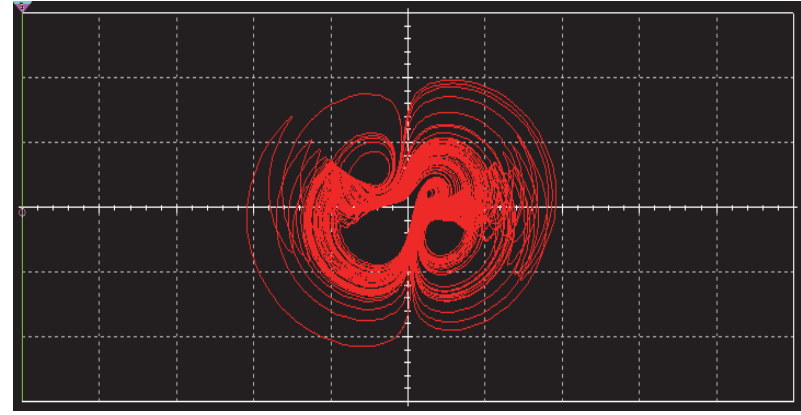

(a)

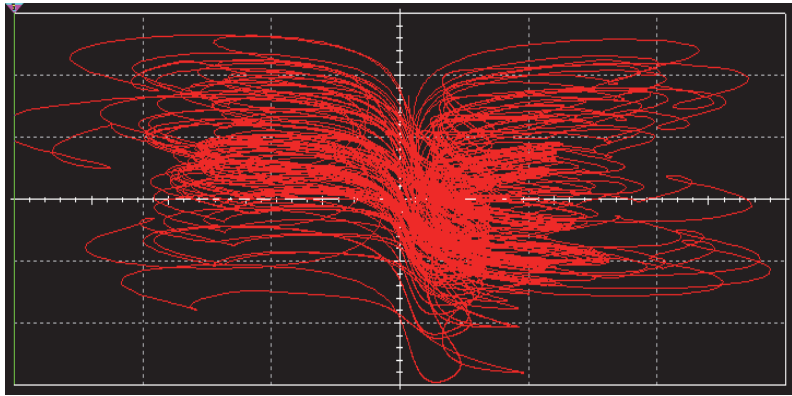

(c)

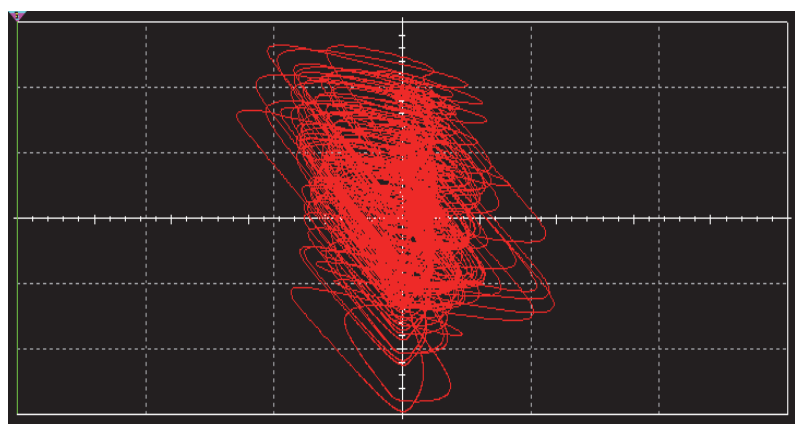

(e)

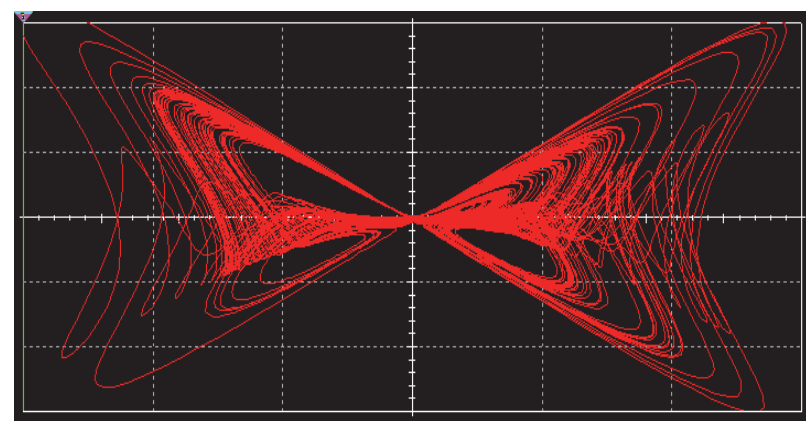

(b)

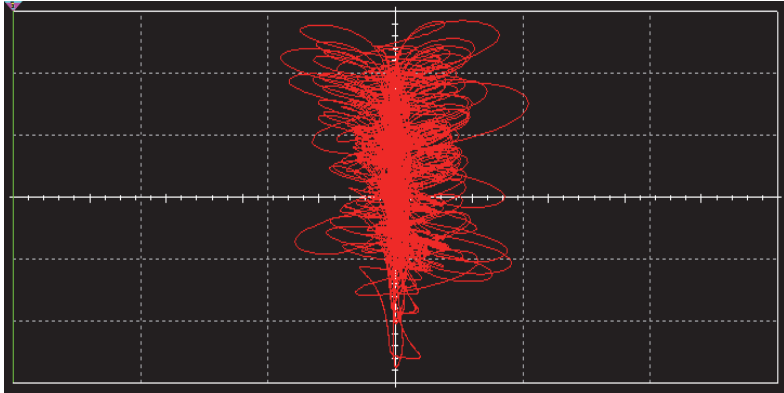

(d)

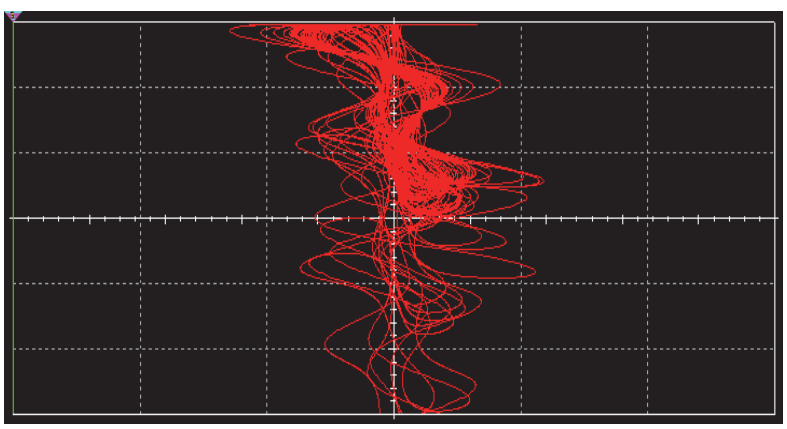

(f)

Figure 6: Multisim implementation of the chaotic System (17): (a) $x-y$ plane with scales $5 \mathrm{~V} / \mathrm{div}$ and $2 \mathrm{~V} / \mathrm{div}$, (b) $x$ - $z$ plane with scales $2 \mathrm{~V} / \mathrm{div}$ and $1 \mathrm{~V} / \mathrm{div}$, (c) $x$ - $w$ plane with scales $2 \mathrm{~V} /$ div and $1 \mathrm{~V} / \mathrm{div}$, (d) $z$ - $w$ plane with scales $2 \mathrm{~V} /$ div and $1 \mathrm{~V} / \mathrm{div}$, (e) $y$ - $w$ plane with scales $2 \mathrm{~V} / \mathrm{div}$ and $1 \mathrm{~V} / \mathrm{div}$, and (f) $y$ - $u$ plane with scales $2 \mathrm{~V} / \mathrm{div}$ and $2 \mathrm{~V} / \mathrm{div}$.

Figure 2 is the simulation verification of synchronization for the master and the slave memristor-based hyperchaotic Systems (21) and (22) when selecting $k^{\prime}=k_{i}=4(i=$ $1 \cdots 5)$, and the systems choose the initial conditions for both systems, respectively, such that $x(0)=4, y(0)=1.2, z(0)=$ $0.5, w(0)=-3.6, u(0)=6$ and $x^{\prime}(0)=-5, y^{\prime}(0)=2$, $z^{\prime}(0)=1, w^{\prime}(0)=-0.8, u^{\prime}(0)=5$. These figures demonstrate the synchronization of the master and slave Systems (21) and (22).

\section{A New Memristor-Based Hyperchaotic System Circuit Implementation}

In this section, modularized design methods will be implemented in the new memristor-based hyperchaotic systems mentioned above. Multisim software is used to generate the circuit simulation results, and the corresponding physical circuit experiments are conducted to verify the hyperchaotic attractor existences of this hyperchaotic system.

(A1) Memristors Circuit Implementation. Figure 3 shows the circuit configuration of memristor inspired by [16].

In this memristor circuit, a factor $0.1 / \mathrm{V}$ multiplier AD633JN is used. Therefore, $m=R_{f} / R_{m}=0.1, n=$ $\left(100 R_{f} / R_{n}\right)(0.01 / V)=0.01$, and

$$
W=\frac{R_{f}}{R_{m}}+3 \cdot \frac{100 R_{f}}{R_{n}}\left(\frac{0.01}{V}\right) \cdot u^{2}
$$

where $R_{f}$ is the feedback resistor of the amplifier. 


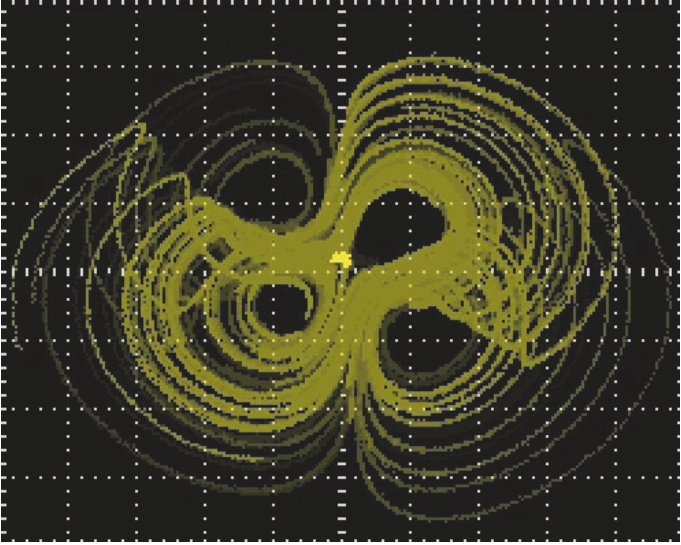

(a)

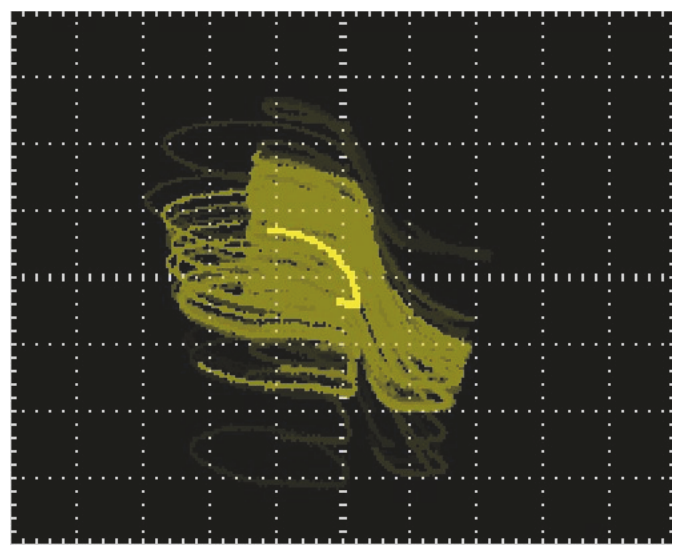

(c)

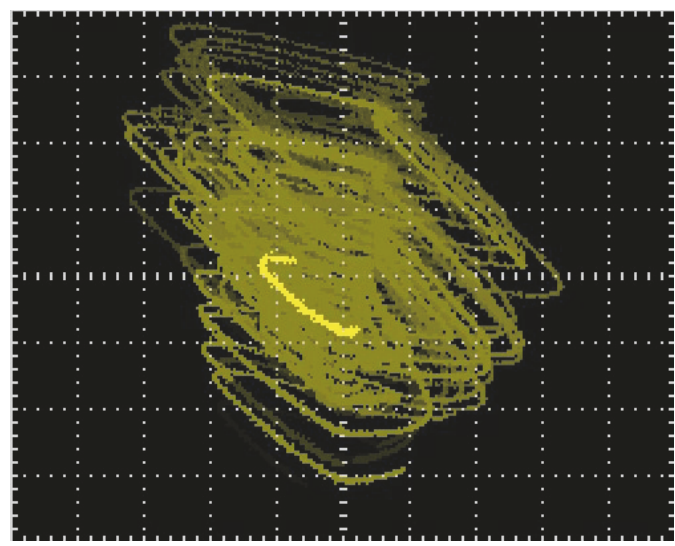

(e)

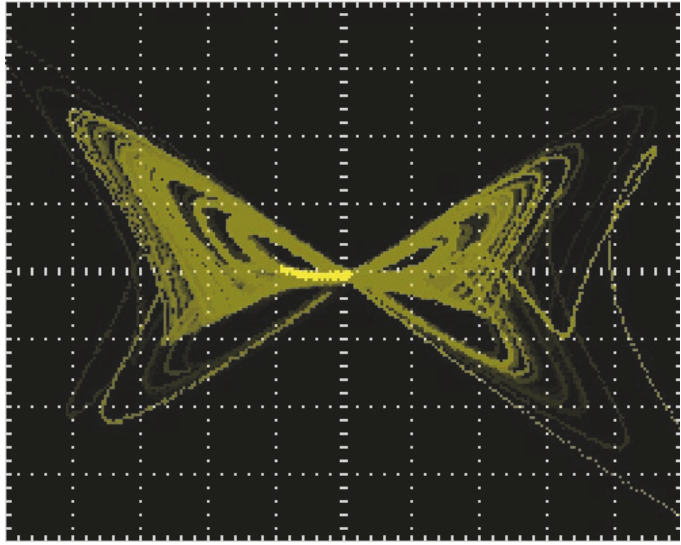

(b)

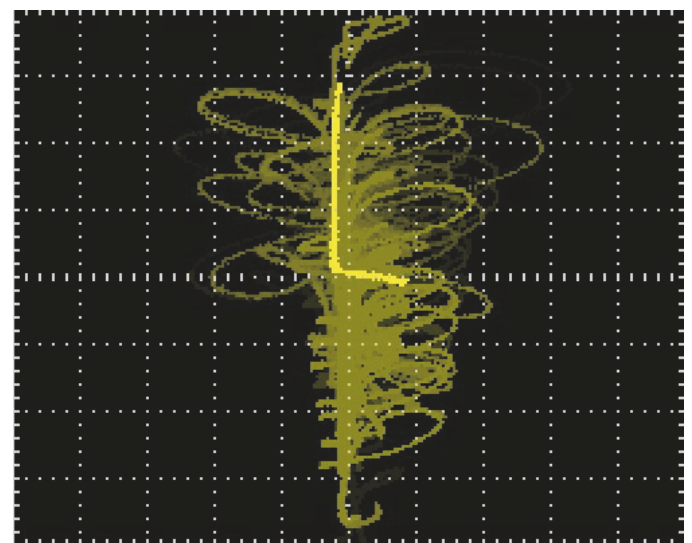

(d)

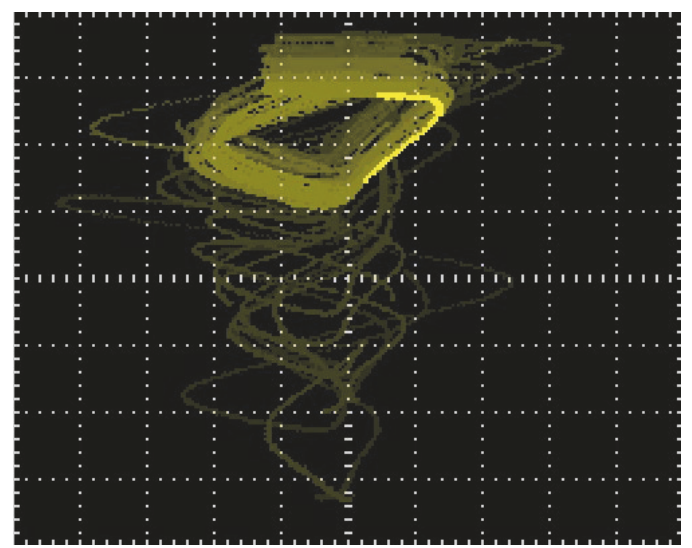

(f)

FIGURE 7: Physical circuit implementation hardware and digital oscilloscope diagrams of the chaotic System (17): (a) $x$ - $y$ plane with scales $2 \mathrm{~V} /$ div and $1 \mathrm{~V} / \mathrm{div}$, (b) $x$ - $z$ plane with scales $2 \mathrm{~V} / \mathrm{div}$ and $1 \mathrm{~V} / \mathrm{div}$, (c) $x-\mathrm{w}$ plane with scales $2 \mathrm{~V} / \mathrm{div}$ and $1 \mathrm{~V} / \mathrm{div}$, (d) $z$ - $w$ plane with scales 1 $V /$ div and $1 \mathrm{~V} / \mathrm{div}$, (e) $y$ - $w$ plane with scales $1 \mathrm{~V} / \mathrm{div}$ and $1 \mathrm{~V} / \mathrm{div}$, and (f) $y$ - $u$ plane with scales $1 \mathrm{~V} / \mathrm{div}$ and $2 \mathrm{~V} / \mathrm{div}$.

Parameter $k$ represents the strength of a memristor.

$$
k=\frac{R_{f}}{m R_{m}}=\frac{R_{f}}{300 n R_{n}}
$$

In Figure 4, select the following resistors and the capacitor, $R_{f}=2 \mathrm{k} \Omega, R_{m}=1 \mathrm{M} \Omega, R_{n}=66 \mathrm{k} \Omega, R_{15}=200 \mathrm{k} \Omega, R_{c}=1 \mathrm{k} \Omega$, $C_{4}=10 n F$.
Figure 4 is the Multisim simulation for the flux-controlled memristor with $x$ input with sinusoid signal. This demonstrates the typical closed loop characteristics of the memristor developed by equation (7) and part of equation (6).

(A2) Memristor-Based Chaotic System Circuit Analysis. The implementation of memristor-based chaotic system uses the 
modularized design method applied on System (17). After the time-scale transformation method is applied on System (17), System (17) becomes System (32).

$$
\begin{aligned}
\frac{d x}{d t}= & -\frac{1}{\tau_{0} R_{1} C_{1}} x-\frac{1}{\tau_{0} R_{2} C_{1}}(-y) \\
& -\frac{1}{10 \tau_{0} R_{3} C_{1}}(-y) z-\frac{1}{10 \tau_{0} R_{4} C_{1}} k x W(u) \\
\frac{d y}{d t}= & -\frac{1}{\tau_{0} R_{5} C_{2}} x-\frac{1}{\tau_{0} R_{6} C_{2}}(-y)-\frac{1}{10 \tau_{0} R_{7} C_{2}} x z \\
& -\frac{1}{\tau_{0} R_{8} C_{2}}(-w) \\
\frac{d z}{d t}= & -\frac{1}{\tau_{0} R_{9} C_{3}} z-\frac{1}{10 \tau_{0} R_{10} C_{3}} x(-y) \\
& -\frac{1}{10 \tau_{0} R_{11} C_{3}} x u-\frac{1}{10 \tau_{0} R_{12} C_{3}} y w \\
\frac{d w}{d t}= & -\frac{1}{\tau_{0} R_{13} C_{4}} y-\frac{1}{10 \tau_{0} R_{14} C_{4}} x(-z) \\
& -\frac{1}{10 \tau_{0} R_{15} C_{4}} z u \\
\frac{d u}{d t}= & -\frac{1}{\tau_{0} R_{16} C_{5}} x
\end{aligned}
$$

where $\tau_{0}=100$.

Therefore,

$$
\begin{aligned}
\frac{d x}{d t}= & -1400 x-1400(-y)-400(-y) z \\
& -100 k x W(u) \\
\frac{d y}{d t}= & -100 x-1600(-y)-100 x z-100(-w) \\
\frac{d z}{d t}= & -7800 z-100 x(-y)-100 x u-100 y w \\
\frac{d w}{d t}= & -1000 y-15 x(-z)-30 z u \\
\frac{d u}{d t}= & -100 x
\end{aligned}
$$

Furthermore, the paper employs the unified compression coefficient, $s=1 / 20$, for each variable in order not to exceed the range of the oscilloscope used to the circuit. Then

$$
\begin{aligned}
& s x \longrightarrow x \\
& s y \longrightarrow y \\
& s z \longrightarrow z \\
& s w \longrightarrow w
\end{aligned}
$$

Substituting (34) into (33), we can obtain

$$
\begin{aligned}
\frac{d x}{d t}= & -1400 x-1400(-y)-8000(-y) z \\
& -100 k x W(u) \\
\frac{d y}{d t}= & -100 x-1600(-y)-2000 x z-100(-w) \\
\frac{d z}{d t}= & -7800 z-100 x(-y)-2000 x u-2000 y w \\
\frac{d w}{d t}= & -1000 y-300 x(-z)-600 z u \\
\frac{d u}{d t}= & -100 x
\end{aligned}
$$

Comparing equations (35) to (32), parameters $R$ and $C$ in the circuit can be calculated when using the factor of 0.1 multiplier. $R_{1}=R_{2}=75 \mathrm{k} \Omega, R_{3}=1 \mathrm{k} \Omega, R_{4}=1 M \Omega, R_{5}=1 M \Omega$, $R_{6}=68 \mathrm{k} \Omega, R_{7}=5.1 \mathrm{k} \Omega, R_{8}=1 \mathrm{M} \Omega, R_{9}=13 \mathrm{k} \Omega, R_{10}=5.1 \mathrm{k} \Omega, R_{11}=$ $R_{12}=1 M \Omega, R_{13}=100 \mathrm{k} \Omega, R_{14}=33.3 \mathrm{k} \Omega, R_{15}=330 \mathrm{k} \Omega, R_{16}=$ $220 \mathrm{k} \Omega$.

In the modularized circuit design for System (17), there are five channels for each variable. The circuit implementation uses analog amplifiers LF347N and AD633JN multipliers to implement the addition and the integral operations. Some additional $R$ and $C$ components are also selected in the circuit implementation. $R_{17}=R_{18}=R_{19}=R_{20}=10 \mathrm{k} \Omega, R_{21}=R_{22}=R_{23}$ $=R_{24}=20 \mathrm{k} \Omega, C_{1}=C_{2}=C_{3}=C_{4}=C_{5}=10 \mathrm{nF}, R_{f}=2.2 \mathrm{k} \Omega$, $R_{c}=1 k \Omega, R_{m}=1 M \Omega, R_{n}=68 \mathrm{k} \Omega$.

The circuit channels for first four variables are shown in Figure 5, and the fifth one is shown in Figure 3.

(A3) Circuit Implementation for the New 5D Chaotic Systems. According to the circuit design, the Multisim simulation implementation for the memristor-based hyperchaotic System (17) is demonstrated in Figure 6. That shows the attractor phases graphs of different planes for System (17).

Attractor phases of System (17) as shown in Figure 6 own similar hyperchaotic characteristics with those of System (5) which are elaborated in [37]. It is known that practical analog devices are not ideal. Therefore, in reality, the voltages added on the amplifiers are not exceeding $\pm 13.5 \mathrm{~V}$ in order to guarantee the system variable outputs do not exceed the amplifier linear region. This paper employs $\pm 12.5 \mathrm{~V}$ across the amplifier in the physical circuits hardware, and the corresponding attractor phases graphs are shown in Figure 7.

Comparing Figure 6 to Figure 7, it is shown that the physical experiment attractor phase results match those conducted by Multisim and demonstrate the existence of the memristorbased hyperchaotic attractor for System (17). Furthermore, the memristor-based Wang hyperchaotic system has similar characteristics with those of the original Wang hyperchaotic system which is verified by the Multisim simulation and physical experimental results. The improved modularized method with compression coefficients is flexible, reliable, and straightforward to realize the physical implementation. The memristor-based hyperchaotic system has potential and 
bright application physically. For example, it can realize the physical communication encryptions more reliably and easily. Future works will focus on the physical applications for communication encryptions by using the memristor-based hyperchaotic system.

\section{Conclusions}

The paper developed a new 5D memristor-based chaotic system with a flux-controlled memristor. The dynamics analysis of the system showed that the new system is a hyperchaotic system. The adaptive controller and update laws for the synchronization of the new system were designed and conducted. Furthermore, the modularized design method for the physical circuit experiment implementation is applied to realize the circuit by Multisim and physical experiments hardware. After the implementation of these circuits, then the comparisons between Multisim simulation and results from the physical experiments showed that these two kinds of results matched with each other and verified the existence of the attractors from the phase plane graphs. Furthermore, this new higher-dimensional chaotic system with memristor owns more complex dynamics and can be applied in a wide range of applications such as encryptions. This is also the future work for this work.

\section{Data Availability}

The data used to support the findings of this study are available from the corresponding author upon request.

\section{Conflicts of Interest}

The authors declare that there are no conflicts of interest regarding the publication of this paper.

\section{Acknowledgments}

This work was supported by grants from the project supported by the Scientific Research Starting Foundation for the Returned Overseas Chinese Scholars, Ministry of Education of China, Tianjin Key Laboratory of Civil Aircraft Airworthiness and Maintenance in CAUC (104003020106) and the National Basic Research Program of China (3122017009).

\section{References}

[1] L. O. Chua, "Memristor-the missing circuit element," IEEE Transactions on Circuit Theory, vol. 18, no. 5, pp. 507-519, 1971.

[2] K. J. Kuhn, "Considerations for ultimate CMOS scaling," IEEE Transactions on Electron Devices, vol. 59, no. 7, pp. 1813-1828, 2012.

[3] D. B. Strukov, G. S. Snider, D. R. Stewart, and R. S. Williams, "The missing memristor found," Nature, vol. 453, pp. 80-83, 2008.

[4] G. Wang, M. Cui, B. Cai, X. Wang, and T. Hu, "A Chaotic Oscillator Based on HP Memristor Model," Mathematical Problems in Engineering, vol. 2015, Article ID 561901, 12 pages, 2015.
[5] L. Chua, "Memristor, hodgkin-huxley, and edge of chaos," Nanotechnology, vol. 24, no. 38, Article ID 383001, 2013.

[6] P. Junsangsri and F. Lombardi, "Design of a hybrid memory cell using memristance and ambipolarity," IEEE Transactions on Nanotechnology, vol. 12, no. 1, pp. 71-80, 2013.

[7] M. R. Azghadi, B. Linares-Barranco, D. Abbott, and P. H. W. Leong, "A Hybrid CMOS-Memristor Neuromorphic Synapse," IEEE Transactions on Biomedical Circuits and Systems, vol. 11, no. 2, pp. 434-445, 2017.

[8] S. H. Jo, T. Chang, I. Ebong, B. B. Bhadviya, P. Mazumder, and W. Lu, "Nanoscale memristor device as synapse in neuromorphic systems," Nano Letters, vol. 10, no. 4, pp. 1297-1301, 2010.

[9] J. Borghetti, G. S. Snider, P. J. Kuekes, J. J. Yang, D. R. Stewart, and R. S. Williams, "Memristive' switches enable "stateful" logic operations via material implication," Nature, vol. 464, no. 7290, pp. 873-876, 2010.

[10] Y. Zhang, Y. Shen, X. Wang, and Y. Guo, "A novel design for memristor-based OR gate," IEEE Transactions Circuits Systems II: Express Briefs, vol. 62, p. 1, 2015.

[11] X. Zhu, X. J. Yang, C. Q. Wu, N. Xiao, J. J. Wu, and X. Yi, "Performing stateful logic on memristor memory," IEEE Transactions on Circuits and Systems II: Express Briefs, vol. 60, no. 10, pp. 682-686, 2013.

[12] Q. Chen, X. Wang, H. Wan, R. Yang, and J. Zheng, "A circuit design for multi-inputs stateful OR gate," Physics Letters A, vol. 380, no. 38, pp. 3081-3085, 2016.

[13] L. Wang and S. Duan, "A chaotic attractor in delayed memristive system," Abstract and Applied Analysis, Art. ID 726927, 7 pages, 2012.

[14] F. Setoudeh, A. Khaki Sedigh, and M. Dousti, "Analysis of a chaotic memristor based oscillator," Abstract and Applied Analysis, vol. 2014, 2014.

[15] B. Muthuswamy, "Implementing memristor based chaotic circuits," International Journal of Bifurcation and Chaos, vol. 20, no. 5, pp. 1335-1350, 2010.

[16] J. Ma, Z. Chen, Z. Wang, and Q. Zhang, "A four-wing hyperchaotic attractor generated from a $4 \mathrm{D}$ memristive system with a line equilibrium," Nonlinear Dynamics, vol. 81, no. 3, pp. 12751288, 2015.

[17] J. Zhang, "Combination-combination hyperchaos synchronization of complex memristor oscillator system," Mathematical Problems in Engineering, vol. 2014, Article ID 591089, 13 pages, 2014.

[18] Z.-H. Lin and H.-X. Wang, "Image encryption based on chaos with PWL memristor in Chua's circuit," in Proceedings of the 2009 International Conference on Communications, Circuits and Systems, ICCCAS 2009, pp. 964-968, USA, July 2009.

[19] M. Itohand and L. O. Chua, "Memristor oscillators," International Journal of Bifurcation \& Chaos, vol. 18, pp. 3183-3206, 2008.

[20] I. Petráš, "Fractional-order memristor-based Chua’s circuit," IEEE Transactions on Circuits and Systems II: Express Briefs, vol. 57, no. 12, pp. 975-979, 2010.

[21] B. Muthuswamy and L. O. Chua, "Simplest chaotic circuit," International Journal of Bifurcation and Chaos, vol. 20, no. 5, pp. 1567-1580, 2010.

[22] H. Li, L. Wang, and S. Duan, "A memristor-based scroll chaotic system-design, analysis and circuit implementation," International Journal of Bifurcation \& Chaos, vol. 24, no. 7, pp. $1-10,2014$. 
[23] D. A. Prousalis, C. K. Volos, I. N. Stouboulos, and I. M. Kyprianidis, 4-D memristive chaotic system with different families of hidden attractors, 2018.

[24] C. Wang, H. Xia, and L. Zhou, "Implementation of a new memristor-based multiscroll hyperchaotic system," Pramana-Journal of Physics, vol. 88, no. 2, 2017.

[25] J. Mou, K. Sun, H. Wang, and J. Ruan, "Characteristic Analysis of Fractional-Order 4D Hyperchaotic Memristive Circuit," Mathematical Problems in Engineering, vol. 2017, Article ID 2313768, 13 pages, 2017.

[26] Y. Li, W. Chi, and X. Huang, "A New Memristor Based Hyperchaotic System," in Proceedings of the 2012 5th International Workshop on Chaos-Fractals Theories and Applications (IWCFTA), pp. 131-135, Dalian, Liaoning, China, October 2012.

[27] W. Shen, Z. Zeng, and G. Wang, "Feedback stabilization of memristor-based hyperchaotic systems," in Proceedings of the 2013 IEEE 3rd International Conference on Information Science and Technology, ICIST 2013, pp. 211-214, China, March 2013.

[28] P. Liu, R. Xi, P. Ren, J. Hou, and X. Li, "Analysis and Implementation of a New Switching Memristor Scroll Hyperchaotic System and Application in Secure Communication," Complexity, vol. 2018, Article ID 3497640, 15 pages, 2018.

[29] V.-T. Pham, C. Volos, and L. V. Gambuzza, "A memristive hyperchaotic system without equilibrium," The Scientific World Journal, vol. 2014, Article ID 368986, 2014.

[30] V. T. Pham, S. Vaidyanathan, C. K. Volos, S. Jafari, and X. Wang, "A chaotic hyperjerk system based on memristive device," in Advances and Applications in Chaotic Systems, Springer International Publishing, 2016.

[31] D. A. Prousalis, C. K. Volos, I. N. Stouboulos, and I. M. Kyprianidis, "Hyperchaotic memristive system with hidden attractors and its adaptive control scheme," Nonlinear Dynamics, vol. 90, no. 3, pp. 1681-1694, 2017.

[32] A. G. Radwan, A. T. Azar, S. Vaidyanathan, J. M. MunozPacheco, and A. Ouannas, "Fractional-Order and Memristive Nonlinear Systems: Advances and Applications," Complexity, vol. 2017, Article ID 3760121, 2 pages, 2017.

[33] J. Ma, L. Wang, S. Duan, and Y. Xu, "A multi-wing butterfly chaotic system and its implementation," International Journal of Circuit Theory and Applications, vol. 45, no. 11, pp. 1873-1884, 2017.

[34] J. Wang, H. Li, R. Wang, L. Wang, and Y. Wang, "A new four-dimensional smooth four-wing hyperchaotic system and its circuit implementation," Journal of Shandong University (Natural Science), vol. 50, no. 11, pp. 104-112, 2015.

[35] S. M. Yu, Chaotic Systems and Choatic Circuits- Principle, Design and its application in communications, XiDian University Press, Xian, 1st edition, 2011.

[36] S. M. Yu, W. K. S. Tang, and G. Chen, "Design and implementation of multi-wing butterfly chaotic attractors via Lorenz-type systems," International Journal of Bifurcation \& Chaos, vol. 20, pp. 29-41, 2010.

[37] R. Wang, H. Sun, J. Wang, L. Wang, and Y. Wang, "Applications of modularized circuit design in a new hyper-chaotic system circuit implementation," Chinese Physics B, vol. 24, Article ID 020501, 2015.

[38] R. Wang, Q. Xie, Y. Huang, H. Sun, and Y. Sun, "Design of a switched hyperchaotic system and its application," International Journal of Computer Applications in Technology, vol. 57, no. 3, p. 207, 2018.
[39] S. Vaidyanathan, V.-T. Pham, C. Volos, and X. Wang, "A Chaotic System with an Infinite Number of Equilibrium Points: Dynamics, Horseshoe, and Synchronization," Advances in Mathematical Physics, vol. 2016, Article ID 4024836, 8 pages, 2016. 


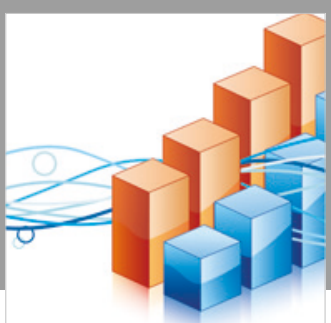

Advances in

Operations Research

\section{-n-m}
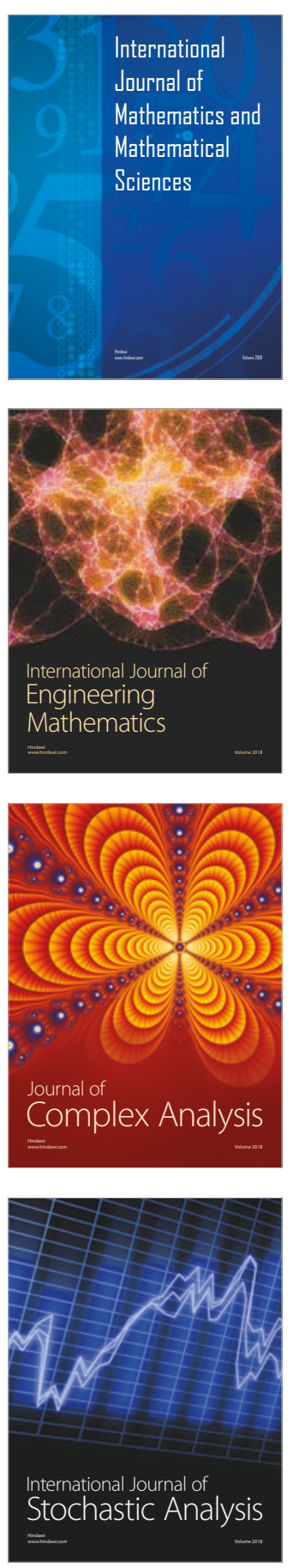
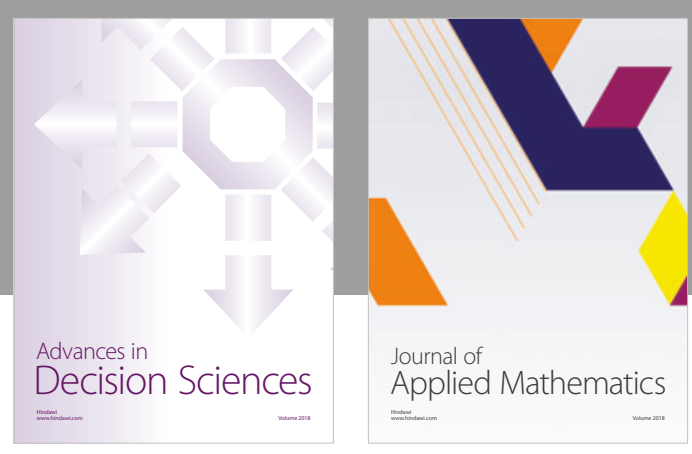

Journal of

Applied Mathematics
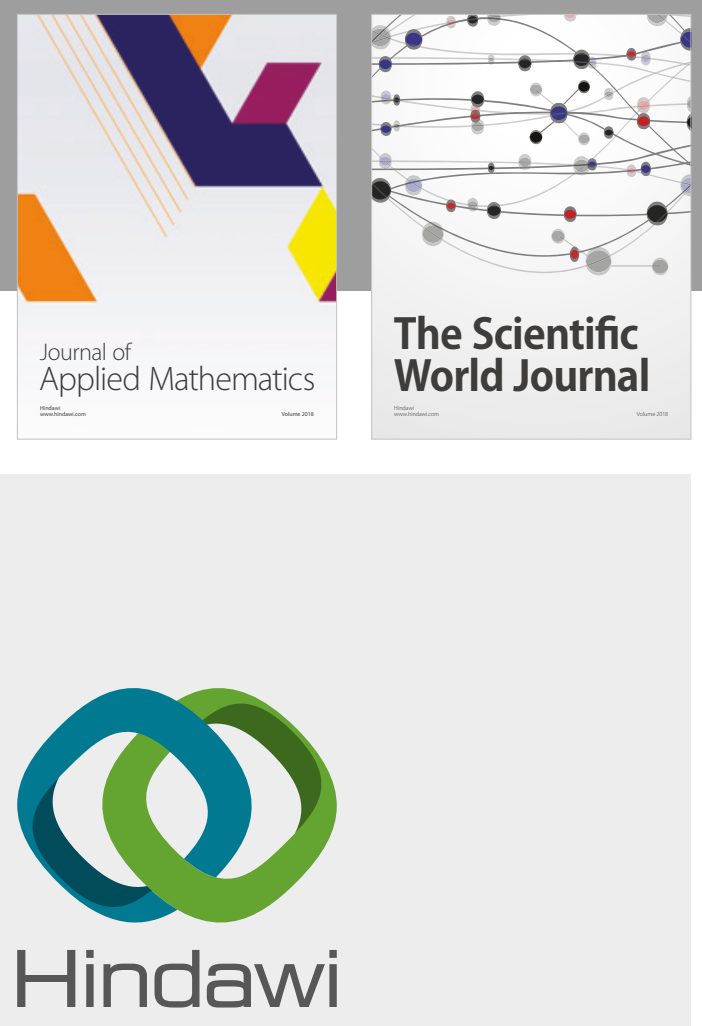

Submit your manuscripts at

www.hindawi.com

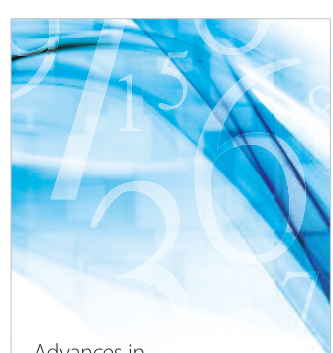

Advances in
Numerical Analysis
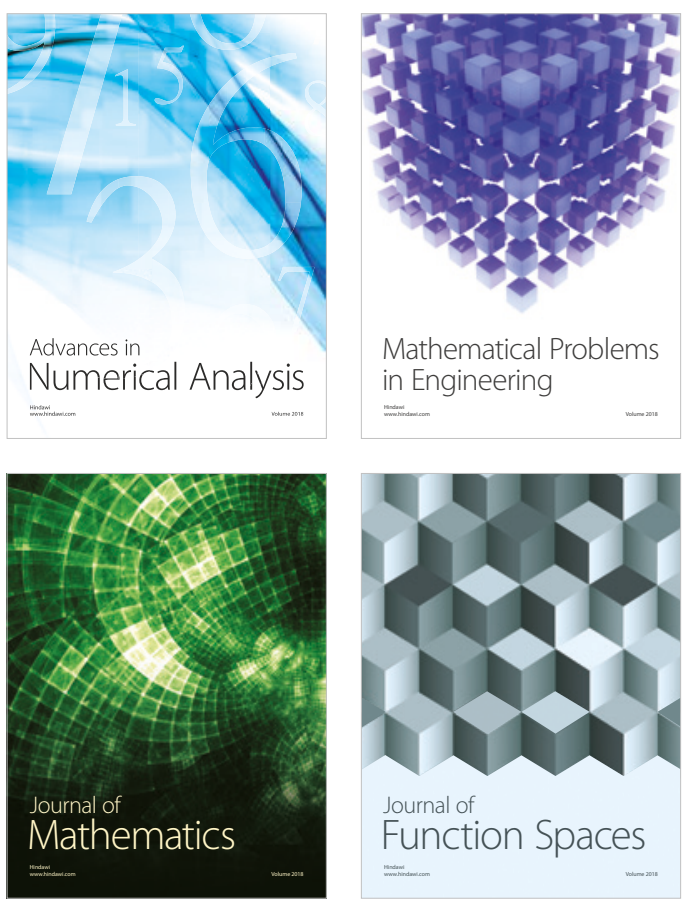

Mathematical Problems in Engineering

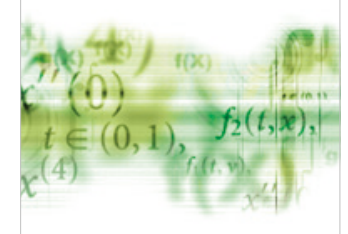

International Journal of

Differential Equations

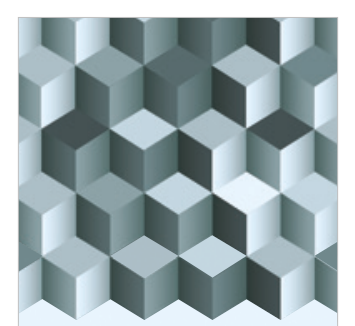

Journal of

Function Spaces
The Scientific

World Journal

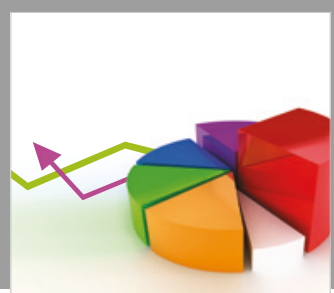

Journal of

Probability and Statistics
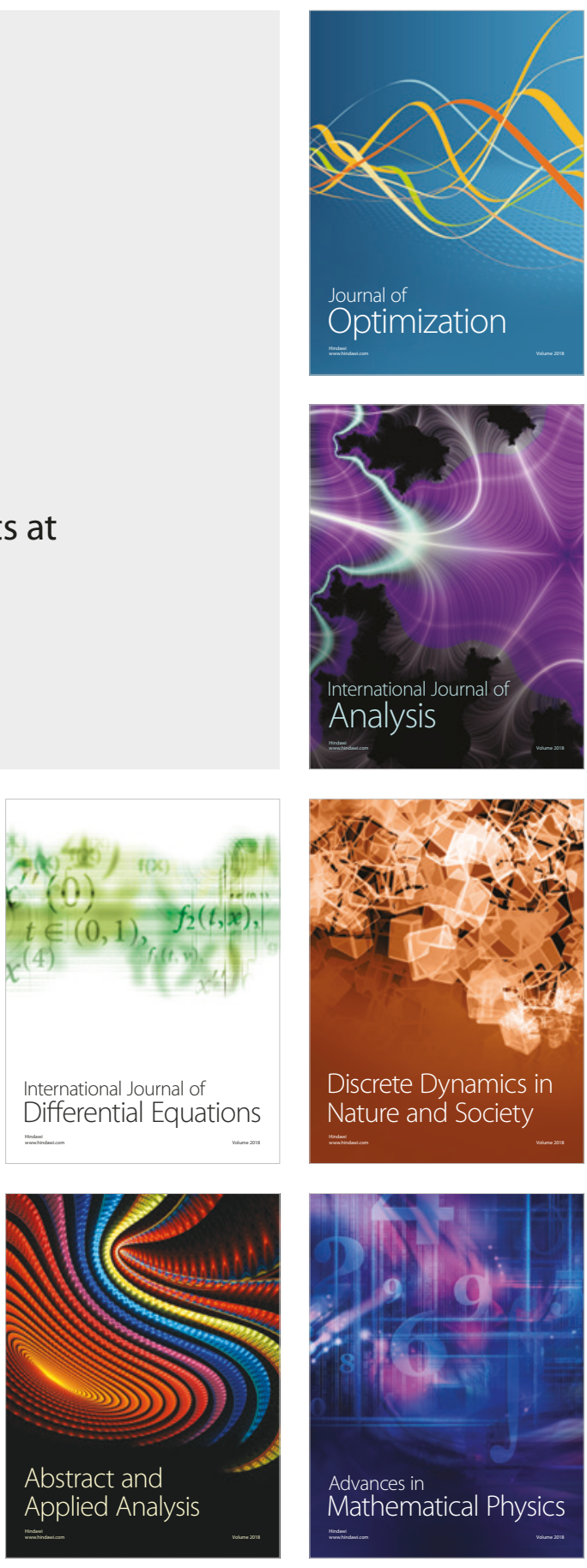